\title{
Elastic Positronium-Atom Scattering Using the Stochastic Variational Method
}

\author{
I. A. Ivanov, ${ }^{1}$ J. Mitroy, ${ }^{1}$ and K. Varga ${ }^{2}$ \\ ${ }^{1}$ Faculty of Science, Northern Territory University, Darwin NT 0909, Australia \\ ${ }^{2}$ Solid State Division, Oak Ridge National Laboratory, Oak Ridge, 37831 Tennessee
}

(Received 9 April 2001; published 24 July 2001)

\begin{abstract}
The stochastic variational method is used in conjunction with stabilization ideas to compute the low energy phase shifts and scattering lengths for positronium-atom scattering. Results are obtained for the Ps-H, Ps- $\mathrm{Li}^{+}$, Ps-He, and Ps-Ps systems. The Ps-H scattering lengths are probably accurate to better than $5 \%$ and are the most accurate so far computed. The results for Ps- $\mathrm{Li}^{+}$and Ps-Ps scattering represent the first published scattering lengths for these systems. The positive scattering length for completely spinaligned ${ }^{3} \mathrm{Ps}-{ }^{3} \mathrm{Ps}$ scattering, namely $2.95 a_{0}$, is particularly significant since it demonstrates the feasibility of forming a stable Bose-Einstein condensate of ${ }^{3} \mathrm{Ps}$ atoms.
\end{abstract}

DOI: 10.1103/PhysRevLett.87.063201

One of the most difficult problems in atomic collision theory is the positronium-atom scattering problem. The source of the difficulty lies in the fact that both the projectile and the target are composite objects with an internal structure. This means that the interaction matrix elements involve multicenter integrals which are difficult and time consuming to evaluate [1]. Particular sources of concern are the evaluation of the exchange matrix element involving the electrons in the target and the electron forming part of the positronium. An even more formidable problem is the inclusion of the van der Waals interaction between the positronium (Ps) projectile and the atomic target. One can point to very few calculations that have included this effect, and even then the calculations were restricted to Ps-H scattering.

In this article, the stochastic variational method [2-5] is applied to the calculation of Ps-atom scattering for the Ps-H, Ps-Li ${ }^{+}$, Ps-He, and Ps-Ps systems. The Ps-H system is probably the best known from the theoretical point of view [6-9]. There have been no scattering calculations of the Ps- $\mathrm{Li}^{+}$system as such, although there have been calculations of the alternate $e^{+}$-Li entrance channel [10]. The Ps-He system has been studied previously by a number of authors and at the moment there is a great deal of variation (a factor of 5) in the threshold cross section according to the different calculations [11-14] and different experiments [15-19]. The present calculation includes all the physical Ps-helium interactions and to a large extent resolves most of the theoretical issues surrounding the Ps-He scattering length. There has been no published calculation of Ps-Ps scattering even though knowledge of the scattering length is of vital importance to proposals to form a stable Bose-Einstein condensate (BEC) of spin-aligned triplet Ps atoms [20,21]. Besides being half comprised of antimatter, a ${ }^{3}$ Ps BEC would also be unique because it could be formed at relatively high temperatures $(\sim 150 \mathrm{~K})$ $[20,21]$.

The method as applied is not a traditional scattering calculation, rather it uses stabilization ideas [6,22-24] to extract the phase shifts from the positive energy pseudo-
PACS numbers: 34.10.+x, 03.75.Fi, 36.10.Dr

continuum that results from the SVM diagonalization of the Hamiltonian. The SVM uses a random search to optimize the exponents of a set of explicitly correlated Gaussians (ECGs) and has the advantage that evaluation of the exchange (and other) matrix elements is easily accomplished [2-5]

The present calculation scheme is now described. Configuration space is divided into two regions, an inner or interaction region and a scattering region. In the inner region, the stochastic variational method (SVM) is used to define an ECG basis (dimension $=K$ ) that gives an accurate solution of the Schrödinger equation for the lowest energy state. For a system such as Ps-H which supports a bound state, this amounts to a standard bound state calculation. However, the Ps-He system does not support a bound state and the procedure must be modified. In this case the exponents, $\alpha_{i}$ of the Gaussians connecting the electron and positron to the nucleus are restricted to be larger than a certain minimum size, say $\alpha_{i}>0.01$. This constrains the electron and positron to be localized reasonably close to the nucleus and results in an SVM iteration procedure that effectively solves the Schrödinger equation in some sort of box.

Once the inner wave function has been obtained, a set of ECGs designed to represent positive energy Ps was added to the basis. First an 8 Gaussian representation of the Ps ground state was constructed (the energy obtained was -0.2499972 hartree). Then a series of ECGs was constructed by multiplying the Ps Gaussians with a Gaussian with the Ps center of mass coordinate as its argument. The exponents of the center of mass Gaussians, $\alpha_{i}$ were chosen to form an even tempered sequence. The ratio between adjacent exponents was approximately 1.3:2.0 and the exponents spanned the range from 1 to $10^{-4}$. The set of the basis functions thus constructed is used to describe relative motion of Ps and the target. For some systems, namely Ps-H and $\mathrm{Ps}_{2}$, the final ECGs consisted of symmetrized products of center of mass Gaussians joining two composite objects (either Ps or $\mathrm{H}$ ) which were also written as a linear combination of ECGs, e.g., for Ps-H 


$$
\Psi_{\text {out }}^{i j k}=\exp \left(-\alpha_{i} R^{2}\right) \psi_{j}^{P s}\left(\mathbf{r}_{0}, \mathbf{r}_{1}\right) \psi_{k}^{H}\left(\mathbf{r}_{2}\right),
$$

where $R$ is the distance between the Ps and $\mathrm{H}$ centers of mass, $\mathbf{r}_{0}$ is the positron coordinate, and the $\mathbf{r}_{i}(i>0)$ are the set of electron coordinates. The $\psi_{j}^{P s}\left(\mathbf{r}_{0}, \mathbf{r}_{1}\right)$ and $\psi_{k}^{H}\left(\mathbf{r}_{2}\right)$ are the Gaussians used in the expansion of the Ps and $\mathrm{H}$ ground states.

The inner and outer basis functions were then checked for linear dependence and ECGs having large overlaps with existing basis functions were excluded, giving a final basis of dimension $M$. The basis was diagonalized by standard techniques and the phase shift information extracted. The essential idea is based on the often made observation $[6,22-24]$ that the positive energy pseudostates give a reasonable representation of the scattering function over a restricted radial range.

Two different approaches were used to determine the phase shifts. In the first, the overlap integral of the Ps ground state with the positive energy pseudostate is computed at a succession of values of $R$, the Ps center of mass coordinate, i.e., the overlap between $\delta\left[\left(\mathbf{r}_{0}+\mathbf{r}_{1}\right) / 2-\mathbf{R}\right] \psi_{P S}\left(\mathbf{r}_{0}, \mathbf{r}_{1}\right)$ and $\Phi\left(\mathbf{r}_{0}, \mathbf{r}_{i}\right)$ was computed. Then a least squares fit over $R \in[10,25] a_{0}$ to $B \sin \left(k R+\delta_{0}\right)$ was used to extract the phase shift.

In the second, the positron-nucleus correlation function defined by

$$
C(x)=4 \pi x^{2} \int d^{3} r_{i} d^{3} r_{0} \delta\left(\mathbf{r}_{0}-\mathbf{x}\right)\left|\Phi\left(\mathbf{r}_{0}, \mathbf{r}_{i}\right)\right|^{2}
$$

is computed for a succession of $x$ values and fitted to $B \sin ^{2}\left(k x+\delta_{0}\right)$ over $x \in[10,25] a_{0}$.

The two approaches of extracting the phase shifts generally gave phase shifts that agreed to about $0.01 \mathrm{rad}$. A useful diagnostic check was to compare the magnitude of the electron-nucleus and positron-nucleus correlation functions over the radial values of interest. They generally agreed to better than $0.1 \%$

The calculations on Ps-H and Ps-Ps scattering were fully $a b$ initio calculations. The fixed core stochastic variational method (FCSVM) was used for the Ps- $\mathrm{Li}^{+}$and Ps-He systems. The FCSVM replaces the full Hamiltonian for the $N_{e}$ electrons and a positron by a model Hamiltonian with the core electrons removed, viz

$$
\begin{aligned}
H= & -\frac{1}{2} \nabla_{0}^{2}-\frac{1}{2} \nabla_{1}^{2}-V_{\mathrm{dir}}\left(\mathbf{r}_{0}\right)+V_{\mathrm{dir}}\left(\mathbf{r}_{1}\right)+V_{p 1}\left(\mathbf{r}_{0}\right) \\
& +V_{p 1}\left(\mathbf{r}_{1}\right)+V_{\mathrm{exc}}\left(\mathbf{r}_{1}\right)-\frac{1}{r_{01}}+V_{p 2}\left(\mathbf{r}_{1}, \mathbf{r}_{0}\right)+\lambda \hat{P} .
\end{aligned}
$$

In this expression, $\mathbf{r}_{1}$ refers to the electron coordinate, while $\mathbf{r}_{0}$ refers to the positron coordinate. The direct potential $\left(V_{\text {dir }}\right)$ for the core is taken from a Hartree-Fock wave function and is the same (although opposite in sign) for the electron and the positron. The exchange potential $\left(V_{\mathrm{exc}}\right)$ between the scattering electron and the Hartree-Fock core was computed exactly. The polarization potential $V_{p 1}$ is defined with the functional form

$$
V_{p 1}(r)=-\frac{\alpha_{d} g^{2}(r)}{2 r^{4}}
$$

The factor $\alpha_{d}$ is the static dipole polarizability of the core and $g^{2}(r)$ is a cutoff function. The same cutoff function was adopted for both the positron and electrons. Its form was chosen as

$$
g^{2}(r)=1-\exp \left(-r^{6} / \rho^{6}\right),
$$

where $\rho$ is an adjustable parameter. The two-body polarization potential $\left(V_{p 2}\right)$ is defined as

$$
V_{p 2}\left(\mathbf{r}_{0}, \mathbf{r}_{1}\right)=\frac{\alpha_{d}}{r_{0}^{3} r_{1}^{3}}\left(\mathbf{r}_{0} \cdot \mathbf{r}_{1}\right) g\left(r_{0}\right) g\left(r_{1}\right)
$$

Inclusion of the two-body potential ensures that the polarization interaction reduces to a van der Waals type interaction when the Ps is at large distances from the nucleus. The $\mathrm{Li}^{+}$core polarization potential was tuned to the spectrum of neutral lithium and was defined with $\alpha_{d}=0.1925 a_{0}^{3}$ and $\rho=1.40 a_{0}$ [5]. The polarization potential $\left(\alpha_{d}=1.383 a_{0}^{3}, \rho=2.40 a_{0}\right)$ for helium was defined by adding it to a static-exchange calculation of $e^{-}$-He scattering and adjusting $\rho$ until the scattering length agreed with the known value of $1.2 a_{0}$ [26].

The operator

$$
\lambda \hat{P}=\sum_{i=1} \lambda\left|\phi_{i}\right\rangle\left\langle\phi_{i}\right|
$$

is an orthogonalizing pseudopotential that acts to produce wave functions orthogonal to the occupied core orbitals when $\lambda[5,27]$ is a large positive number. It $(\lambda)$ was set to $10^{5}$ hartree for the present calculations.

The Ps-H system has two different electron spin states with only the singlet electron spin configuration having a bound state (the bound PsH ground state). For the spinsinglet case, the PsH inner wave function had $K=450$ with an energy of -0.789190 hartree. The triplet case which does not have a bound state had $K=350$. The final dimensions were $M=1083$ and $M=968$ for the singlet and triplet cases, respectively.

Figure 1 shows the phase shifts for both triplet and singlet Ps-H scattering. Also shown in Fig. 1 is an effective range fit to the phase shift using the expression

$$
\tan \left(\delta_{0}\right)=-A k \text {, }
$$

where $A$ is the scattering length. The phase shifts have minor fluctuatation about the effective range fit since the positive energy pseudostates do not give an exact representation of the scattering wave function despite giving an approximate representation over a limited radial range.

The SVM scattering lengths of $4.3 a_{0}$ and $2.2 a_{0}$ are close to converged and broadly compatible with the 25 year old estimates of Drachman and Houston [6]. (They deduced a singlet scattering length of $4.5 a_{0}$ from a stabilization calculation with corrections to compensate for their relatively 


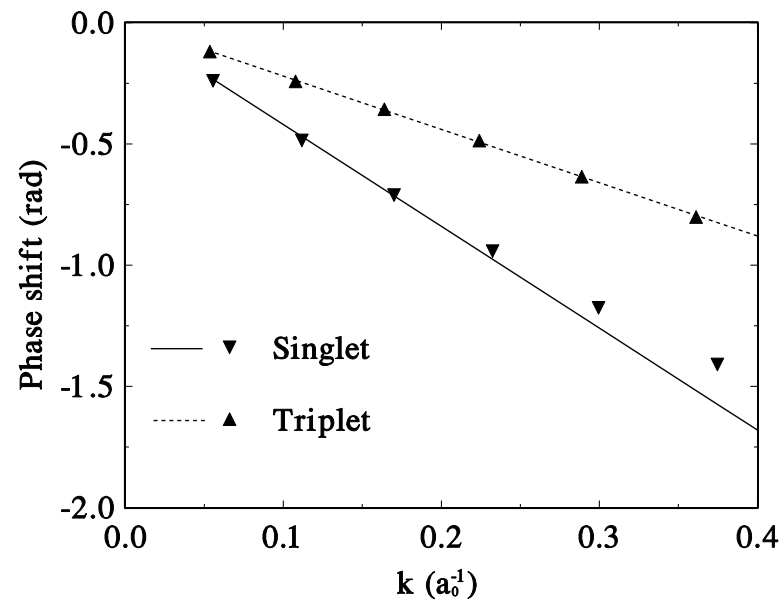

FIG. 1. The $s$-wave phase shifts for Ps-H scattering as a function of $k$ (in $a_{0}^{-1}$ ). Phase shifts (modulo $\pi$ ) for both the singlet spin configuration and triplet configuration are shown. The lines represent effective range fits to the phase shifts.

small basis size). The most recent Kohn variational estimate of $3.5 a_{0}$ [9] is susceptible to systematic errors since a severely truncated partial wave expansion was used to evaluate some of the matrix elements [9].

The scattering calculation for Ps- $\mathrm{Li}^{+}(K=170, M=$ 305) was based upon an existing wave function for the $\mathrm{Ps}_{-} \mathrm{Li}^{+}$ground state $[5,28]$. The phase shifts shown in Fig. 2 were not computed by fitting to $\sin (k R+\delta)$. Instead the fit was made to an asymptotic function that included the polarization of the Ps atom due to the field of the residual $\mathrm{Li}^{+}$ion. The scattering length of $12.9 a_{0}$ is $25 \%$ larger than the scattering length of $10.1 a_{0}$ derived from the Ps- $\mathrm{Li}^{+}$binding energy $[5,28]$ using effective range theory.

The Ps-He scattering calculations ( $K=240, M=469)$ were done for two different core potentials and the phase shifts are displayed in Fig. 3. In the first calculation, the core-polarization potentials were omitted. This calculation allows for direct and exchange interaction of the elec-

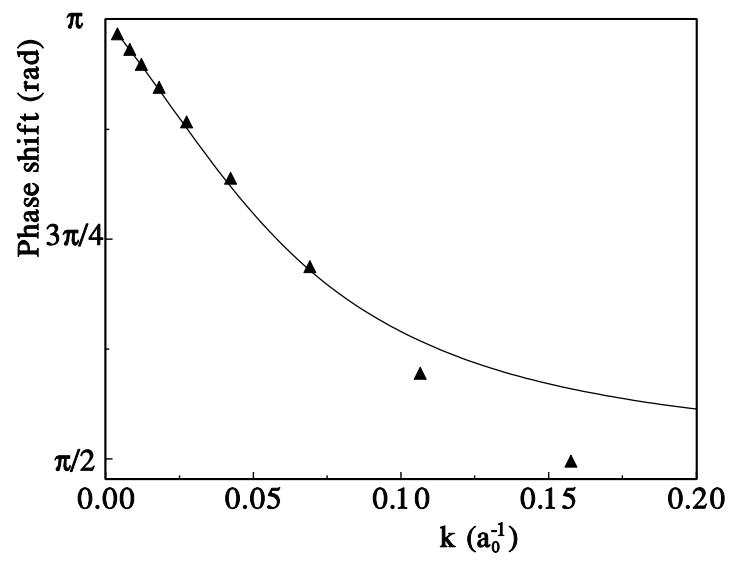

FIG. 2. The $s$-wave phase shifts for $\mathrm{Ps}_{\mathrm{si}}-\mathrm{Li}^{+}$scattering as a function of $k$ (in $a_{0}^{-1}$ ). The solid line represents an effective range fit [with an additional $\pi \alpha_{d} k^{2} / 3$ term added to Eq. (8)] to the phase shift. tron and positron with the $\mathrm{He}$ atom, but does not allow for any distortion of the He atom during the scattering event. The scattering length for this calculation was $1.82 a_{0}$, in agreement with the 22 state $R$-matrix calculation which has the same physical content [12]. Inclusion of the polarization interactions leads to a modest reduction in the magnitude of the phase shifts and therefore in the cross section. Although the polarization potential was derived semiempirically, the same FCSVM approach was used to compute the binding energies of $\mathrm{PsHe}^{+}$[25] and $\mathrm{PsLi}^{+}$ $[5,28]$ ground states, giving energies in very good agreement with the $a b$ initio calculations $[5,25,28]$. The present calculation with polarization uses a Hamiltonian that is more realistic than any previously used, and the threshold cross section $10.4 \pi a_{0}^{2}$, strongly favors the larger estimates of the threshold cross section, namely $8.4 \pm 0.9$ [17], 9.0 [19], and $13 \pm 4 \pi a_{0}^{2}$ [18]. A recent smaller estimate of the cross section, $2.6 \pm 0.5 \pi a_{0}^{2}$ at $1 \mathrm{eV}$ energy [16] by the Michigan group is effectively excluded. Although there is some uncertainty in the precise value of the scattering length, using $\rho=1.50 a_{0}$ (derived from the $e^{+}$-helium scattering length) would give a threshold cross section, $8.6 \pi a_{0}^{2}$, that was still consistent with the larger measurements. The present calculations resolve current controversy $[11,12,16,18]$ about the size of Ps-He scattering length.

Phase shifts for the Ps-Ps system were obtained for two spin configurations. They were for the configuration with both electrons and both positrons in a singlet state $(K=350, M=1026)$, and the ${ }^{3}$ Ps- ${ }^{3}$ Ps configuration $(K=350, M=893)$ with all spins aligned. The ${ }^{1}\left(2 e^{-}\right)-{ }^{1}\left(2 e^{+}\right)$configuration has a bound state (the $\mathrm{Ps}_{2}$ molecule) and the binding energy with the inner basis was 0.0160034 hartree (best binding energy $=0.0160037$ [3]). Both sets of phase shifts shown in Fig. 4 vary smoothly with energy and the derived scattering lengths have relatively small uncertainties. The present ${ }^{3} \mathrm{Ps}-{ }^{3} \mathrm{Ps}$

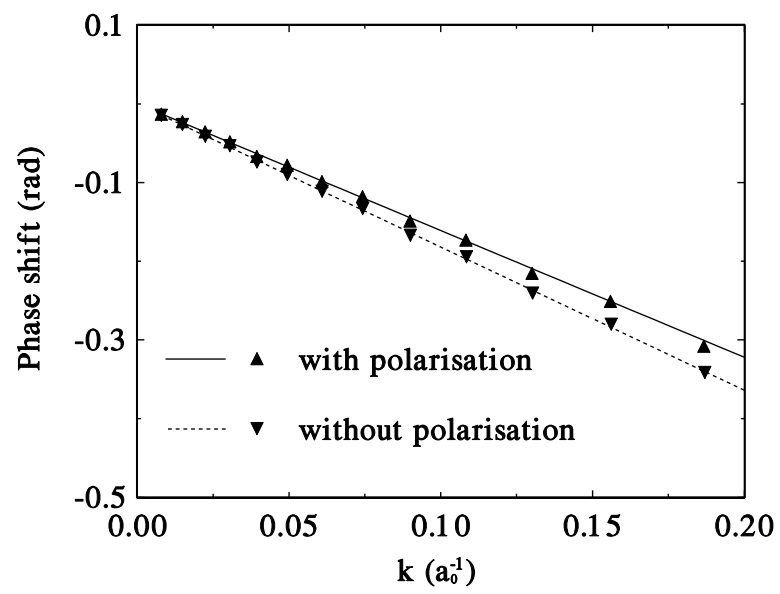

FIG. 3. The Ps-He $s$-wave phase shifts for model potentials with and without polarization potentials as a function of $k$ (in $a_{0}^{-1}$ ). The lines show effective range fits to the phase shifts. 


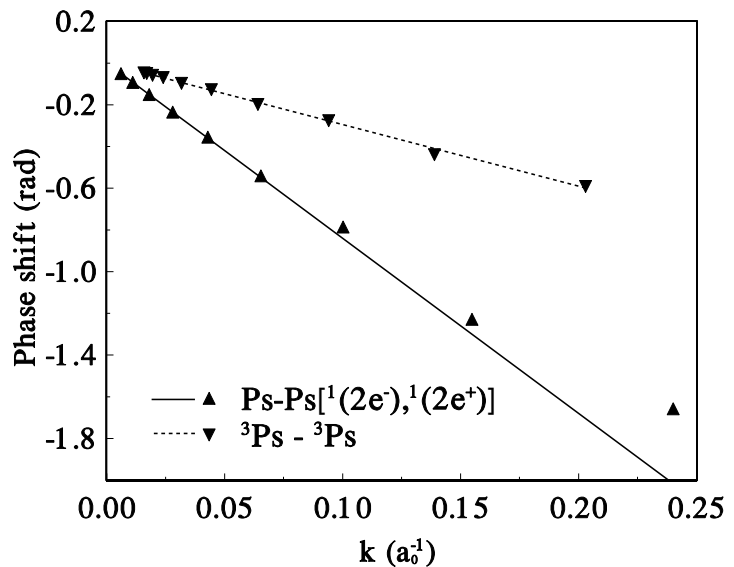

FIG. 4. The $s$-wave phase shifts (modulo $\pi$ ) for Ps-Ps $\left[{ }^{1}\left(2 e^{-}\right),{ }^{1}\left(2 e^{+}\right)\right]$and the spin aligned ${ }^{3}$ Ps- ${ }^{3}$ Ps scattering as a function of $k$ (in $a_{0}^{-1}$ ). The lines are effective range fits to the phase shifts.

scattering length is close to the back of the envelope estimate of the ${ }^{3} \mathrm{Pss}^{3} \mathrm{Ps}$ scattering length used by Platzman and Mills [20], namely $\sim 2 a_{0}$, in their analysis of the feasibility of making a BEC out of Ps atoms. The positive value of the ${ }^{3} \mathrm{Ps}-{ }^{3} \mathrm{Ps}$ scattering length, namely $2.95 a_{0}$, demonstrates the possibility of forming a stable BEC of ${ }^{3}$ Ps atoms.

Most of the scattering lengths listed in Table I are close to converged. Variations in the size of the inner wave function basis for all systems resulted in only minor changes in the derived scattering lengths. It is estimated that the uncertainties in the derived scattering lengths are in the $2 \%-5 \%$ range. While the present application of the SVM has been used to solve some previously intractable problems in Ps scattering, the SVM can also be sensibly applied to investigate other difficult scattering problems with composite particles, e.g., meson-nucleon or nucleon-nucleon scattering in constituent quark models or exciton-exciton scattering in semiconductors.

[1] H. W. S. Massey and C. B. O. Mohr, Proc. Phys. Soc. London 67, 695 (1954).

[2] V. I. Kukulin and V. M. Krasnopolsky, J. Phys. G 3, 795 (1977).

[3] Y. Suzuki and K. Varga, Stochastic Variational Approach to Quantum-Mechanical Few-Body Problems (Springer, New York, 1998).

[4] K. Varga and Y. Suzuki, Phys. Rev. C 52, 2885 (1995); Comput. Phys. Commun. 106, 157 (1997).

[5] G. G. Ryzhikh, J. Mitroy, and K. Varga, J. Phys. B 31, 3965 (1998).

[6] R. J. Drachman and S. K. Houston, Phys. Rev. A 12, 885 (1975); 14, 894 (1976).

[7] B. A. P. Page, J. Phys. B 9, 1111 (1976).
TABLE I. Scattering lengths (in $a_{0}$ ) for a number of Ps-atom scattering systems. The effective range theory (ERT) values were derived from the binding energy $(E)$ using $A=1 / \sqrt{2 \mu E}$ where $\mu$ is the system reduced mass.

\begin{tabular}{lccc}
\hline \multicolumn{1}{c}{ System } & Present & ERT & Other \\
\hline Ps-H $(S=0)$ & 4.3 & 2.5 & $4.5^{\mathrm{a}}, 5.20^{\mathrm{b}}, 3.49^{\mathrm{c}}, 5.84^{\mathrm{d}}$ \\
Ps-H $(S=1)$ & 2.2 & & $2.36^{\mathrm{a}}, 2.45^{\mathrm{b}}, 2.46^{\mathrm{c}}, 2.32^{\mathrm{d}}$ \\
Ps-Li ${ }^{+}$ & 12.9 & 10.1 & \\
Ps-He & 1.61 & & $1.82^{\mathrm{b}}, 1.39^{3}, 0.88^{\mathrm{f}}$ \\
Ps-Ps $\left[{ }^{1}\left(2 e^{-}\right),{ }^{1}\left(2 e^{+}\right)\right]$ & 8.4 & 5.6 & \\
${ }^{3}$ Ps- ${ }^{-P s}$ & 2.95 & & \\
\hline \hline
\end{tabular}

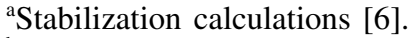

b22 state $R$-matrix calculations $[8,12]$.

${ }^{\mathrm{c} K o h n}$ variational method [9].

${ }^{\mathrm{d}}$ Kohn variational method [7].

${ }^{\mathrm{e}}$ Kohn variational method with model exchange [13].

fThree-state close coupling with model exchange [11].

[8] C. P. Campbell, M. T. McAlinden, F. G. R. S. MacDonald, and H. R. J. Walters, Phys. Rev. Lett. 80, 5097 (1998).

[9] S. K. Adhikari and P. Mandal, J. Phys. B 34, L187 (2001).

[10] K. P. Sarkar, M. Basu, and A. P. Ghosh, J. Phys. B 21, 1649 (1988); M. T. McAlinden, A. A. Kernoghan, and H. R. J. Walters, J. Phys. B 30, 1543 (1994).

[11] P. K. Biswas and S. K. Adhikari, Phys. Rev. A 59, 363 (1999).

[12] J.E. Blackwood, C. P. Campbell, M. T. McAlinden, and H. R. J. Walters, Phys. Rev. A 60, 4454 (1999).

[13] R. J. Drachman and S. K. Houston, J. Phys. B 3, 1657 (1970).

[14] P. A. Fraser, J. Phys. B 1, 1006 (1968).

[15] A. J. Garner, G. Larrichia, and A. Ozen, J. Phys. B 29, 5961 (1996).

[16] M. Skalsey, J. J. Engbrecht, R. K. Bithell, R. S. Vallery, and D. W. Gidley, Phys. Rev. Lett. 80, 3727 (1998).

[17] K. F. Canter, J. D. McNutt, and L. O. Roellig, Phys. Rev. A 12, 375 (1975).

[18] Y. Nagashima, T. Hyodo, F. Fujiwara, and I. Ichimura, J. Phys. B 31, 329 (1998).

[19] P. G. Coleman, S. Rayner, F. M. Jacobsen, M. Charlton, and R. N. West, J. Phys. B 27, 981 (1994).

[20] P. M. Platzman and A. P. Mills, Jr., Phys. Rev. B 49, 454 (1994).

[21] New Directions in Antimatter Chemistry and Physics, edited by C. M. Surko and F. A. Gianturco (Kluwer, The Netherlands, 2001).

[22] F.E. Harris, Phys. Rev. Lett. 19, 173 (1967); 19, 885 (1967).

[23] A. U. Hazi and H. S. Taylor, Phys. Rev. A 1, 1109 (1970).

[24] A. T. Stelbovics and T. Winata, Aust. J. Phys. 43, 485 (1990).

[25] G. G. Ryzhikh and J. Mitroy, J. Phys. B 31, 3465 (1998); 32, 4051 (1999).

[26] R. K. Nesbet, Phys. Rev. A 20, 58 (1979).

[27] V. M. Krasnopolsky and V. I. Kukulin, Sov. J. Nucl. Phys. 20, 470 (1975); J. Mitroy and G. G. Ryzhikh, Comput. Phys. Commun. 123, 103 (1999).

[28] J. Mitroy and G. G. Ryzhikh, J. Phys. B 32, L621 (1999). 